\title{
Analysis on the Influence Factors of Accident Severity: Evidence from Urban River- crossing Tunnels in Shanghai of China
}

\author{
Wan He ${ }^{1}$, Zhuoye Zhang ${ }^{1}$, Linjun $\mathrm{Lu}^{1, *}$ and Zhenyu Wang ${ }^{2}$ \\ ${ }^{1}$ School of Naval Architecture, Ocean and Civil Engineering, Shanghai Jiao Tong University, Shanghai 200240, China \\ ${ }^{2}$ Center for Urban Transportation Research, University of South Florida, Tampa, FL 33601, United States
}

Received 23 August 2018; Accepted 30 October 2018

\begin{abstract}
To analyze various factors influencing the accident severity of urban river-crossing tunnels, 12 influence factors were selected according to the three traffic elements of vehicle, road, and environment, including accident-involved vehicle type, tunnel length, tunnel speed limit, accident occurrence time, and weather. These factors were based on the historical data of 14 urban river-crossing tunnels in Shanghai. A binary logistic regression model was also utilized to identify significant influence factors and analyze the influence degrees of their accident severity. Significance analysis reveals that accident occurrence place, accident-involved vehicle type, tunnel speed limit, number of vehicles involved, and accident type are significantly correlated with accident severity. Moreover, VISSIM traffic simulation model and conflict simulation analysis software called surrogate safety assessment model were utilized to simulate different urban rivercrossing tunnel scenes. The simulation explored the influence degrees of traffic volume, tunnel longitudinal slope, tunnel speed limit, and ratio of heavy trucks, which stand for three traffic elements (i.e., vehicle, road, and environment, respectively) on accident severity. Results show that the factors exerting the greatest influences on accident severity are traffic volume, number of vehicles involved, accident occurrence place, and accident type, followed by tunnel longitudinal slope, tunnel speed limit, and accident-involved vehicle type. Traffic volume, number of vehicles involved, accident type, tunnel longitudinal slope, and accident-involved vehicle type have a significantly positive correlation with accident severity, whereas accident occurrence position and tunnel speed limit have a significantly negative correlation with accident severity. The study results provide a new idea for researches on urban river-crossing tunnels and provide basis and method for tunnel entrance design, speed limit adjustment and regulation of traffic volume.
\end{abstract}

Keywords: Urban river-crossing tunnel, Logistic, Accident severity, Traffic conflict

\section{Introduction}

As important transportation facilities for developing cities along rivers in China and other countries, urban rivercrossing tunnels eliminate obstacles for urban development and satisfy people's daily traffic demand. However, relative to highways and bridges, drivers must adapt to darkness and light when entering and leaving tunnels. During this process, drivers may make wrong judgments and cause traffic accidents. Compared with highway tunnels, longitudinal slopes exist at the entrance and exit of urban river-crossing tunnels. Thus, drivers face road alignment by combining longitudinal slopes and horizontal curves when driving, bringing enormous hidden dangers to traffic. Data released by the World Health Organization (WHO) indicate that approximately 125,000 people die in road traffic accidents (WHO, 2015) every year, and traffic accident has become one of the primary causes of deaths, injuries, and disabilities. Owing to the sealed and special traffic environment of urban river-crossing tunnels, disposing and evacuating once an accident occurs are difficult, resulting in enormous risk to both person and property. Thus, studying traffic accidents in urban river-crossing tunnels is important. The severity of

*E-mail address: linjunlu@situ.edu.cn

ISSN: $1791-2377$ @ 2018 Eastern Macedonia and Thrace Institute of Technology. All rights reserved. doi:10.25103/jestr.115.12 traffic accidents in urban river-crossing tunnels is influenced by driving environmental conditions, such as road conditions, tunnel speed limit, tunnel length, traffic volume, and weather. Collision conditions, such as accident type, accidentinvolved vehicle type, and collision time and place, also influence such tunnels [1-3]. The foundation for effective accident control and prevention is to identify and determine significant risk factors of traffic accident severity in urban river-crossing tunnels and analyze why these factors can exert influences and their mutual effects.

Recent researches from China and other countries on tunnel traffic safety have concentrated on highway tunnels $[4,5]$, but only a few studies regarding urban river-crossing tunnels have been conducted. Direct and indirect evaluation methods are mainly adopted in traffic safety research, in which the former needs mass data based on historical accident data. However, direct evaluation method faces problems such as difficult accident data acquisition, long data accumulation time, and restricted data size [6, 7]. Indirect evaluation method uses alternative indexes to evaluate traffic accidents. Most recent studies utilize traffic conflict technology (TCT), which combines conflict simulation analysis software (SSAM) and microcosmic traffic simulation software (VISSIM) to perform conflict analysis and thus achieve the evaluation goal of traffic safety. 
TCT can be an effective supplement when accident data are lacking [8, 9]. In addition, few studies regarding accident severity have paid attention to the joint influences of various factors. With most existing research on tunnel traffic safety focused on highway tunnels, discussions about urban rivercrossing tunnels are therefore lacking. Few research methods have combined direct and indirect evaluation methods to carry out traffic safety research. However, the attention directed toward the joint effect of influence factors is insufficient. Thus, based on the historical accident data of 14 urban river-crossing tunnels already completed in Shanghai, the influence factors of accident severity in urban rivercrossing tunnels were selected in this study according to the three traffic elements of car, road, and environment. First, a binary logistic model was used to identify significant influence factors and analyze their degrees of influence on accident severity and the joint influences of various factors. Second, the influence degrees of traffic volume, tunnel longitudinal slope, tunnel speed limit, and ratio of heavy trucks on accident severity were further studied by using VISSIM traffic simulation model and SSAM software. Consequently, pertinent risk control and regulation countermeasures of urban river-crossing tunnels were proposed. These factors have great practical significance in reducing accident occurrence rate and lowering accident severity.

\section{State of the art}

Studies from China and other countries focus on highway tunnels when investigating tunnel accident severity. Only a few studies are conducted on urban river-crossing tunnels. Amundsen and Ranes [10] divided a highway tunnel into seven segments after a statistical analysis of 587 highway tunnels. The study found that accident rate is the highest at the tunnel entrance, and tunnel accident severity is higher than highway accident severity. However, accident data derived from written records and reports from the police station lack accuracy. Mashimo [11] described the current status of highway tunnel safety technology in Japan and found through accident statistics that traffic accident rate inside highway tunnels is lower than that outside. However, once an accident occurs in such tunnels, especially a fire disaster, accident severity can be higher than that outside the tunnels. Therefore, Mashimo [11] argued that accident occurrence rate and accident severity can be lowered by optimizing tunnel design and traffic organization and setting ancillary facilities. The study also suggested that emergency safety and fire-fighting facilities should be reinforced. However, the feasibility of the optimization scheme was not verified through calculation or simulation. Lemke [12] analyzed accidents in 68 highway tunnels in Germany. Statistical results showed that road shoulder can affect property loss during accidents, and a two-way lane is the most significant influence factor of tunnel accident severity. Through a statistical analysis of historical accident data of 50 tunnels in Australia, Nussbaumer [13] also found that accident severity inside tunnels is evidently higher than accident severity on open roads. The accident severity of two-lane tunnels was generally higher than that of singlelane tunnels, and the accident rate at tunnel entrances was high. Relative highway and urban river-crossing tunnels have horizontal curves superposed with longitudinal slopes. However, an understanding of the effect of horizontal curves and longitudinal slopes on accident severity in highway tunnels is lacking in previous studies.

In terms of the influence factors of accident severity, existing literature has mainly used mathematical statistics method based on accident data and simulation-based TCT to analyze the relationships between traffic accident severity and influence factors. The influence degrees of traffic safety factors are also determined. Sam et al. [14] conducted a statistical analysis of bus accident data from 2011 to 2015 and studied factors influencing bus accident severity. Results showed that weekend, nighttime conditions, poor pavement behaviors (bending, humid, and rough), and drunk driving present a significant correlation with bus accident severity. However, the accident data may be deficient or insufficient, resulting in errors of result analysis. On the basis of accident data in Palermo, Italy, Potoglou et al. [15] used a hybrid logistic regression model to study the influence factors of accident severity in non-fatal urban road traffic. The study determined that speeding, driver's age, and season are the main factors influencing accident severity. Young drivers also experienced serious traffic accidents more easily than old drivers. In addition, severe accidents occurred more in summer than in winter, but cross-over effects among factors were not considered. Based on a 10-year historical accident data in Wyoming (2002-2011), Ahmed et al. [16] used Bayes binary Logit model to perform a classified study on severe and non-severe accidents of heavy trucks. Results showed that if a heavy truck accident occurs on an interstate expressway, the occurrence probability of a severe accident can increase by 4.5 times. When the field of view was unclear, the pavement was slippery, or in rainy and snowy days, accident severity would significantly increase. However, only the influences of single factors were considered. The joint effects of these factors were not analyzed. Haynes et al. [17] conducted a simulated study of the relationship between traffic accident severity and geometric design parameters of roads. Results showed that slope, lane width, and shoulder breadth can significantly influence traffic accident severity. Jung and Soyoung et al. [18] studied the influences of rainy weather on multi-vehicle collision frequency and accident severity from three dimensionalities of rainfall intensity, water film depth, and insufficient car-following distance. The authors verified the influences of these dimensionalities on traffic operation through VISSIM. The study found that daily rainfall and wind velocity can exert significant influences on accident frequency and accident severity, respectively. However, simulated scenes were few and incomprehensive.

To sum up, while recent studies on tunnel accident severity focused on highway tunnels, few discussed urban river-crossing tunnels. The main research methods are accident data-based statistical analysis method and TCT based on simulation and alternative indexes. However, few studies combined the two methods to analyze accident severity. In addition, most studies on the influence factors of accident severity analyzed the influences of single factors on accident severity, but only a few considered the joint influences of two factors on accident severity.

In view of such deficiencies in existing studies, the influence factors of accident severity in urban river-crossing tunnels were studied in this research. Based on the historical accident data of 14 urban river-crossing tunnels completed in Shanghai, a binary logistic model was initially used to identify the significant influence factors of accident severity that were selected according to three traffic elements of car, road, and environment. Then, the influence degrees of 
various factors and joint effects of every two factors on accident severity were analyzed. An orthogonal test array was also used to design the driving scenes in urban rivercrossing tunnels, and VISSIM traffic simulation model and SSAM conflict simulation software were used for simulation. Moreover, the influences of traffic volume, longitudinal slope, tunnel speed limit, and ratio of heavy trucks on accident severity were further studied. On this basis, pertinent suggestions over the design and management of urban river-crossing tunnels were proposed. These factors can have an important practical significance to lessen accident severity.

The remainder of this study is organized in the following manner. Section 3 expounds the binary logistic model, VISSIM traffic simulation software, SSAM conflict simulation analysis software, and orthogonal test method. Section 4 presents the result analysis. Section 5 summarizes this study and gives relevant conclusions.

\section{Methodology}

\subsection{Selection of influence factors}

Accident data derived from the historical traffic accident data of 14 urban river-crossing tunnels were used. These data were released on the official platform of "110" alarmreceiving centers of Shanghai Public Security Bureau in 2011, an accident database of urban river-crossing tunnels which mainly includes information such as accident severity, accident occurrence time and place, accident form, and accident-involved car type. In the accident data of urban river-crossing tunnels, 365 cases $(2.9 \%)$ caused fatal injuries, 4,533 (36.3\%) caused severe injuries, 6,327 (50.7\%) caused minor injuries, and 1,256 ones $(10.1 \%)$ did not cause any injury. Owing to the evident imbalance among all kinds of data, fatal and severe injuries were combined into "severe injury," which accounted for $39.2 \%$ of the total data. Minor and no injuries were combined into "minor injury," which accounted for $60.8 \%$ of the total data. Twelve influence factors are selected from the three traffic elements of car, road, and environment, namely, accident-involved vehicle type and number of vehicles involved (car); maximum longitudinal slope, minimum radius of horizontal curve, and tunnel length (road); accident occurrence time, whether it happened on a workday, accident occurrence place, weather, number of one-way lanes, tunnel speed limit, and collision type (environment). Table 1 shows the classification and interpretation of variables.

Table 1. Descriptions of Variables

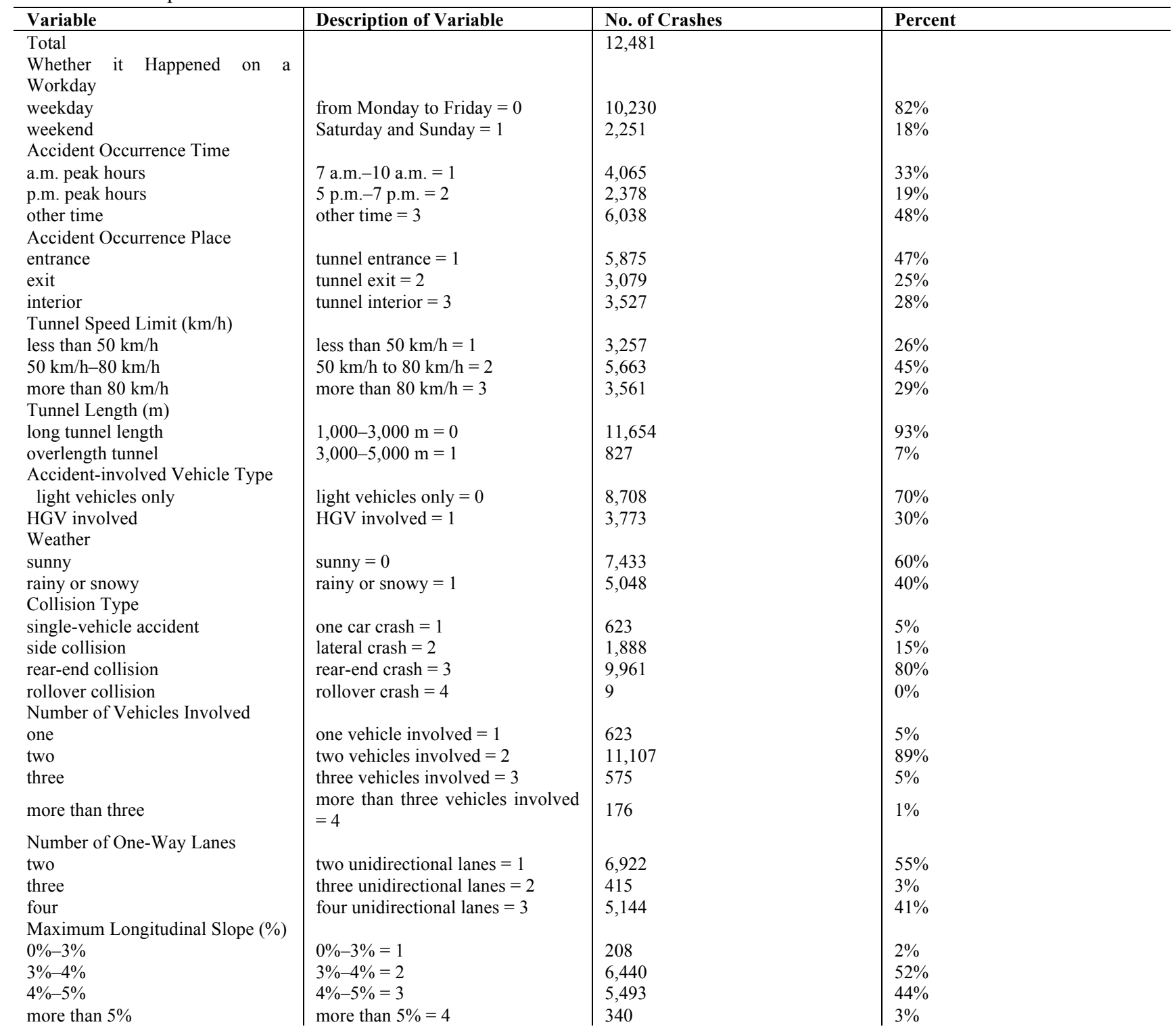


Minimum Radius of Horizontal

Curve (m)

$0-1,000 \mathrm{~m}$

$1,000-2,000 \mathrm{~m}$

$2,000-3,000 \mathrm{~m}$

$3,000-4,000 \mathrm{~m}$

minor injury

severe injury
Injury Severity Level

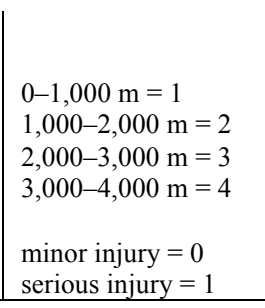

\subsection{Modeling and simulation}

\subsubsection{Binary logistic regression model}

As a mature variable statistical analysis method, logistic regression does not need linear correlation among variables in the model. Covariance matrixes of variables are equal, and residual errors present normal distribution. Thus, the model analysis can be objective. Logistic regression has also been extensively applied to study the influences of all kinds of indexes on traffic safety. The regression can identify key elements influencing accident severity and their degrees of influence $[19,20]$. According to the accident database of urban river-crossing tunnels, accident severity is taken as the dependent variable $y$ in this study. If a person is severely or fatally injured in the $i$ (th) accident, then $y=1$, otherwise $y=0$. Therefore, $\mathrm{t}$ influence factors are correlated with dependent variable $y$ and recorded as $X=\left(x_{1}, x_{2}, \ldots, x_{t}\right)$. Then, under $t$ influence factors, the occurrence probability of an accident that can cause severe or fatal injuries, is $p$; otherwise, $1-p$.

$$
P=\left\{y_{i}=1 \mid \mathrm{X}\right\}=\frac{e^{\beta_{0}+\beta_{1} x_{1}+\beta_{2} x_{2}+\ldots+\beta_{m} x_{m}}}{1+e^{\beta_{0}+\beta_{1} x_{1}+\beta_{2} x_{2}+\ldots+\beta_{m} x_{m}}}
$$

where $x_{i}(i=1,2, \ldots, t)$ is an influence factor of the accident severity in urban river-crossing tunnels. This factor can be a continuous variable, classified variable, or virtual variable. $\beta_{0}$ is a constant term, $\beta_{i}(i=1,2, \ldots, t)$ is the regression coefficient.

\subsubsection{VISSIM and SSAM}

VISSIM can help in the analysis of many traffic problems. For instance, it can reveal influences of road geometrical features, traffic volume, vehicle constitution, and timedependent signal change at an intersection. VISSIM can then output data information needed in the study. SSAM can calculate the number of conflicts and identify conflict types according to vehicle trajectory files output by VISSIM to indirectly realize safety evaluation goals.

The 14 urban river-crossing tunnels in Shanghai selected in this study are all two-hole and four-lane tunnels. The model is established by taking Renmin Road river-crossing tunnel as an example. The tunnel length is $3,097 \mathrm{~m}$, and the distance between the two sides of the tunnel is approximately $1,800 \mathrm{~m}$. Wiedemann 74 car-following model is adopted for traffic simulation, which is mainly applicable to urban road traffic. Simulation time is set as $3,600 \mathrm{~s}$, simulation accuracy is five steps per second, the number of random seeds ranges from 1 to 50 , and simulation speed is $10 \mathrm{~s}$. Influenced by the "black hole" effect, the driver must adapt to sudden brightness change when entering the tunnel. Drivers must slow down near the tunnel entrance because of the longitudinal slope and horizontal curve superposition features of the tunnel. After a simulation of a highway tunnel entranced segment in a mountainous area, Chen [21] found

\begin{tabular}{l|l} 
& \\
4,050 & $32 \%$ \\
8,223 & $66 \%$ \\
0 & $0 \%$ \\
208 & $2 \%$ \\
7,583 & \\
4,898 & $61 \%$ \\
& $39 \%$
\end{tabular}

the laws of the driver entering the tunnel (Fig. 1). Point $\mathrm{P}$ is the tunnel entrance, then the driver starts decelerating at point $A$ and ends deceleration at point $B$ after adaptation. In VISSIM, the deceleration effect can be realized through the "deceleration zone" and the "expected speed decision point".

\subsubsection{Orthogonal test method}

Orthogonal test method refers to an experiment conducted according to an orderly orthogonal array and then the overall test design is completed for final statistical analysis. Orthogonal test design method selects a proper quantity of representative points among many test points and arranges for testing and data analysis based on the existing orthogonal array. The array can realize balanced sampling within the indication range so that each test can have strong representativeness. Orthogonal test method can realize test objectives well under a low-testing frequency, and thus provides great convenience for the study. The orthogonal test scheme is explained as follows:

Step 1) Determine the test indexes: Traffic volume, speed limit, ratio of heavy trucks, and slope are selected as test indexes in this study according to the simulation features of VISSIM and the deficiencies of historical accident data indexes.

Step 2) Determine the factor level: After establishing the Renmin Road river-crossing tunnel model in this study through VISSIM, traffic volume (vehicles per hour, vph), speed limit (kilometer per hour, $\mathrm{km} / \mathrm{h}$ ), ratio of heavy trucks $(\%)$, and longitudinal slope $(\%)$ are properly divided into five grades according to the historical accident data (Table 2).

Step 3) Select the orthogonal array: A proper orthogonal array is selected according to the number of selected factors and factor levels. In this research, four factors are examined, and each factor has five levels. Thus, $L_{25}\left(5^{6}\right)$ (Table 3 ) is selected as the orthogonal test array.

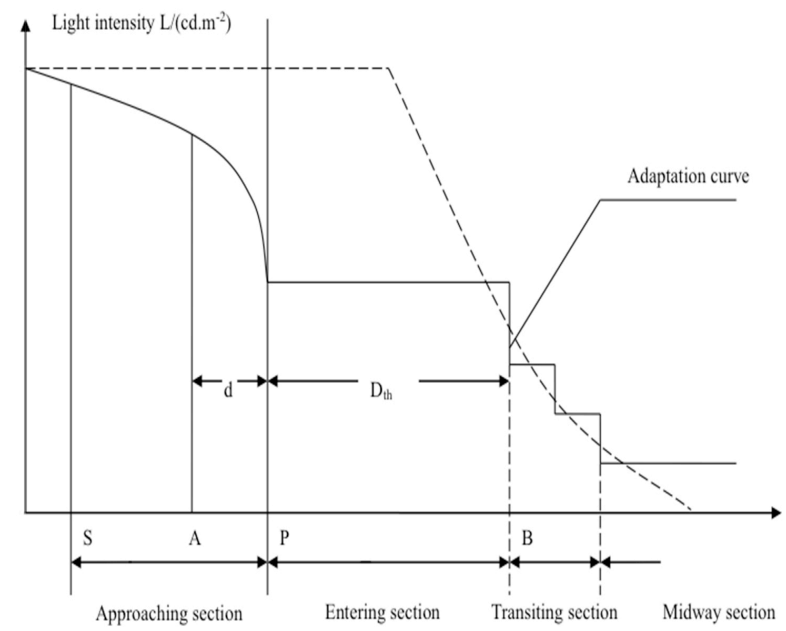

Fig. 1. Process of a vehicle in a tunnel 
Table 2. Classification of Indicators in Orthogonal Test

\begin{tabular}{c|c|c|c|c}
\hline Indicator & $\begin{array}{l}\text { Tunnel } \\
\text { Speed Limit } \\
(\mathbf{k m} / \mathbf{h})\end{array}$ & $\begin{array}{l}\text { Ratio of } \\
\text { Heavy } \\
\text { Trucks (\%) }\end{array}$ & $\begin{array}{l}\text { Traffic } \\
\text { Flow } \\
\text { (vph) }\end{array}$ & $\begin{array}{l}\text { Longitudinal } \\
\text { Slope (\%) }\end{array}$ \\
\hline 1 & 30 & 0 & 1,000 & 2 \\
\hline 2 & 40 & $5 \%$ & 1,500 & 3 \\
\hline 3 & 50 & $10 \%$ & 2,000 & 4 \\
\hline 4 & 60 & $15 \%$ & 2,500 & 5 \\
\hline 5 & 70 & $20 \%$ & 3,000 & 6 \\
\hline
\end{tabular}

Table 3. Orthogonal Test Array $L_{25}\left(5^{6}\right)$

\begin{tabular}{|c|c|c|c|c|c|c|}
\hline $\begin{array}{c}\text { Test } \\
\text { Number }\end{array}$ & $\begin{array}{l}\text { Tunnel } \\
\text { Speed } \\
\text { Limit } \\
(\mathbf{k m} / \mathbf{h})\end{array}$ & $\begin{array}{c}\text { Ratio } \\
\text { of } \\
\text { Heavy } \\
\text { Trucks } \\
(\%)\end{array}$ & $\begin{array}{c}\text { Traffic } \\
\text { Flow } \\
\text { (vph) }\end{array}$ & $\begin{array}{l}\text { Longitudinal } \\
\text { Slope }(\%)\end{array}$ & & \\
\hline 1 & 1 & 1 & 1 & 1 & 1 & 1 \\
\hline 2 & 1 & 2 & 2 & 2 & 2 & 2 \\
\hline 3 & 1 & 3 & 3 & 3 & 3 & 3 \\
\hline 4 & 1 & 4 & 4 & 4 & 4 & 4 \\
\hline 5 & 1 & 5 & 5 & 5 & 5 & 5 \\
\hline 6 & 2 & 1 & 2 & 3 & 4 & 5 \\
\hline 7 & 2 & 2 & 3 & 4 & 5 & 1 \\
\hline 8 & 2 & 3 & 4 & 5 & 1 & 2 \\
\hline 9 & 2 & 4 & 5 & 1 & 2 & 3 \\
\hline 10 & 2 & 5 & 1 & 2 & 3 & 4 \\
\hline 11 & 3 & 1 & 3 & 5 & 2 & 4 \\
\hline 12 & 3 & 2 & 4 & 1 & 3 & 5 \\
\hline 13 & 3 & 3 & 5 & 2 & 4 & 1 \\
\hline 14 & 3 & 4 & 1 & 3 & 5 & 2 \\
\hline 15 & 3 & 5 & 2 & 4 & 1 & 3 \\
\hline 16 & 4 & 1 & 4 & 2 & 5 & 3 \\
\hline 17 & 4 & 2 & 5 & 3 & 1 & 4 \\
\hline 18 & 4 & 3 & 1 & 4 & 2 & 5 \\
\hline 19 & 4 & 4 & 2 & 5 & 3 & 1 \\
\hline 20 & 4 & 5 & 3 & 1 & 4 & 2 \\
\hline 21 & 5 & 1 & 5 & 4 & 3 & 2 \\
\hline 22 & 5 & 2 & 1 & 5 & 4 & 3 \\
\hline 23 & 5 & 3 & 2 & 1 & 5 & 4 \\
\hline 24 & 5 & 4 & 3 & 2 & 1 & 5 \\
\hline 25 & 5 & 5 & 4 & 3 & 2 & 1 \\
\hline
\end{tabular}

Orthogonal test method is used to design 25 experimental scenes. Vehicle trajectory files under different experimental scenes are output through parameter adjustment in VISSIM. These files are then imported to SSAM to obtain a corresponding number of traffic conflicts and conflict types. The analysis method of experimental results obtained through the orthogonal test method can be divided into range analysis method and analysis of variance (ANOVA). The range analysis method is abbreviated as $\mathrm{R}$ method and includes two steps, calculation and judgment. The sum, namely, the $K_{j i}$ of experimental results of factors in the $j$ (th) column corresponding to the $i$ (th) level and average $K_{j i}$ value, namely, the $\bar{K}_{j i}$ of factors in each column at different levels, are calculated. Optimal levels of all factors can be determined according to $K_{j i}$ value and factor properties. Their optimal levels are put together to form an optimal combination of these factors. $R_{j}$ is the difference between the maximum and minimum values of the average index of factors in the $j$ (th) column under different levels, namely, the range.

$$
R_{j}=\max \left(\bar{K}_{j 1}, \bar{K}_{j 2}, \ldots, \bar{K}_{j i}\right)-\min \left(\bar{K}_{j 1}, \bar{K}_{j 2}, \ldots, \bar{K}_{j i}\right)
$$

$R_{j}$ index reflects the changing amplitude of the test indexes of a factor when any of its levels is changed.
Therefore, the greater the $R_{j}$ value, the greater the influence of this factor on test indexes. The influence degree of each factor on test indexes can be judged. Primary and secondary relations of the factors can be determined according to the $R_{j}$ value. The range analysis method is concise, simple, popular, and easy to understand with convenient operation. However, the method neglects test errors. Thus, range analysis method can determine the significance of influence relations. Then, the ANOVA method is introduced.

ANOVA can compensate for the deficiencies of the range analysis method. The change amplitude of test indexes is partially due to the change of factor levels and test errors. The calculation steps of ANOVA are as follows:

Step 1)

Calculate the quadratic sum $s$ of factor deviations:

$k=\frac{1}{n}\left(k_{1}+k_{2}+\ldots+k_{n}\right)=\frac{1}{n} \sum_{i=1}^{n} k_{i}$

$S=\sum_{i=1}^{n}\left(k_{i}-k\right)^{2}$

where $k$ is the arithmetic average of one group of test indexes. The quadratic sum of deviations is the quadratic sum of the difference value between each test index and arithmetic average.

\section{Step 2)}

Calculate the degree $f$ of freedom and the quadratic sum $\bar{s}$ of average deviation of each factor.

$f=n-1$

$\bar{S}=\frac{S}{n-1}$

where $n$ is the number of levels.

Step 3)

Calculate the variance ratio $F$ of each factor.

$F_{j}=\frac{S_{j} / f_{j}}{S_{e} / f_{e}}$

where $S_{i}$ and $f_{i}$ are the quadratic sum of deviations and the degree of freedom of $j$ (th) column, respectively; and $S_{e}$ and $f_{e}$ are the quadratic sum of deviations and the degree of freedom of error columns, respectively (error column is a blank column).

\section{Step 4)}

Significance test

After calculation, the variance ratio of each column is compared with the standard deviation $F_{A}$. Thus, the significance degree of each factor can be obtained. The greater the $F$ value, the more significant the influence of this factor on test indexes. When $F>F_{0.01}, F_{0.01}>F>F_{0.05}$, and $F_{0.05}>F>F_{0.10}$, these factors have significant influences 
on test results. When $F<F_{0.10}$, a separate discussion should be conducted.

\section{Analysis of results and discussion}

\subsection{Analysis of binary logistic model}

Considering the historical accident data of urban rivercrossing tunnels in Shanghai, Stata software was used in this study for logistic regression analysis of the influence factors of accident severity. Table 4 presents the regression results. Results of the test model showed that the $\mathrm{P}$ value of the accident data of the binary logistic model in parallel line test is 0.256 , which is greater than 0.05 . Thus, the model is suitable for studying the accident severity in urban rivercrossing tunnels. In addition, the $\mathrm{P}$ value in the model-fitting information satisfies $0.000<0.05$, indicating that the established model is of statistical significance. The area of the receiver operating characteristic curve (ROC) in the binary logistic model is 0.8317 , which is greater than 0.7 , thereby showing that the result is of certain accuracy. Therefore, the overall degree of fitting of this model is favorable, and influence degrees and directions of various factors on accident severity can be analyzed through regression results.

Table 4 reveals that five significant influence factors are identified through the logistic regression model among the influence factors selected from three traffic elements of car, road, and environment, such as accident occurrence place, accident-involved vehicle type, tunnel speed limit, number of vehicles involved, and accident type. Among these factors, accident occurrence place, the number of vehicles involved, and accident type have the most significant influences on accident severity, followed by tunnel speed limit and accident-involved vehicle type. Moreover, accident-involved vehicle type, number of vehicles involved, and accident type have significantly positive influences on accident severity. By contrast, accident occurrence place and tunnel speed limit have significantly negative influences on accident severity.

The influence of each type under each index on accident severity can also be obtained through the model (Table 5). A concrete analysis is indicated below

Table 4. Various Parameters of the Influencing Factors of the Binary Logistic Model

\begin{tabular}{c|c|c|c}
\hline Variable & Coefficient & $\mathbf{P}>|\mathbf{Z}|$ & {$[\mathbf{9 5 \%}$ Confidence Interval] } \\
\hline Accident Occurrence Place & -1.532901 & 0 & {$[-1.59415,-1.471652]$} \\
Accident-involved Vehicle Type & 0.1668276 & 0.011 & {$[0.0375737,0.2960815]$} \\
Accident Occurrence Time & 0.0408644 & 0.113 & {$[-0.0097221,0.091451]$} \\
Whether it Happened on a Workday & 0.056008 & 0.337 & {$[-0.0582189,0.170235]$} \\
Weather & -0.0060197 & 0.894 & {$[-0.0943444,0.0823049]$} \\
Tunnel Length & -0.0360599 & 0.775 & {$[-0.2834845,0.2113647]$} \\
Number of One-Way Lanes & 0.0233482 & 0.656 & {$[-0.079414,0.1261104]$} \\
Tunnel Speed Limit & -0.150925 & 0.006 & {$[-0.258472,-0.043378]$} \\
Number of Vehicles Involved & 0.4077811 & 0 & {$[0.2695354,0.5460269]$} \\
Collision Type & 1.728475 & 0 & {$[1.608003,1.848947]$} \\
Maximum Longitudinal Slope & 0.0246564 & 0.756 & {$[-0.1305803,0.1798931]$} \\
Minimum Radius of Horizontal Curve & 0.095279 & 0.211 & {$[-0.1154124,0.3059704]$} \\
cons & -3.710401 & 0 & {$[-4.238317,-3.182484]$} \\
\hline
\end{tabular}

Table 5. Regression Results of the Binary Logistic Model

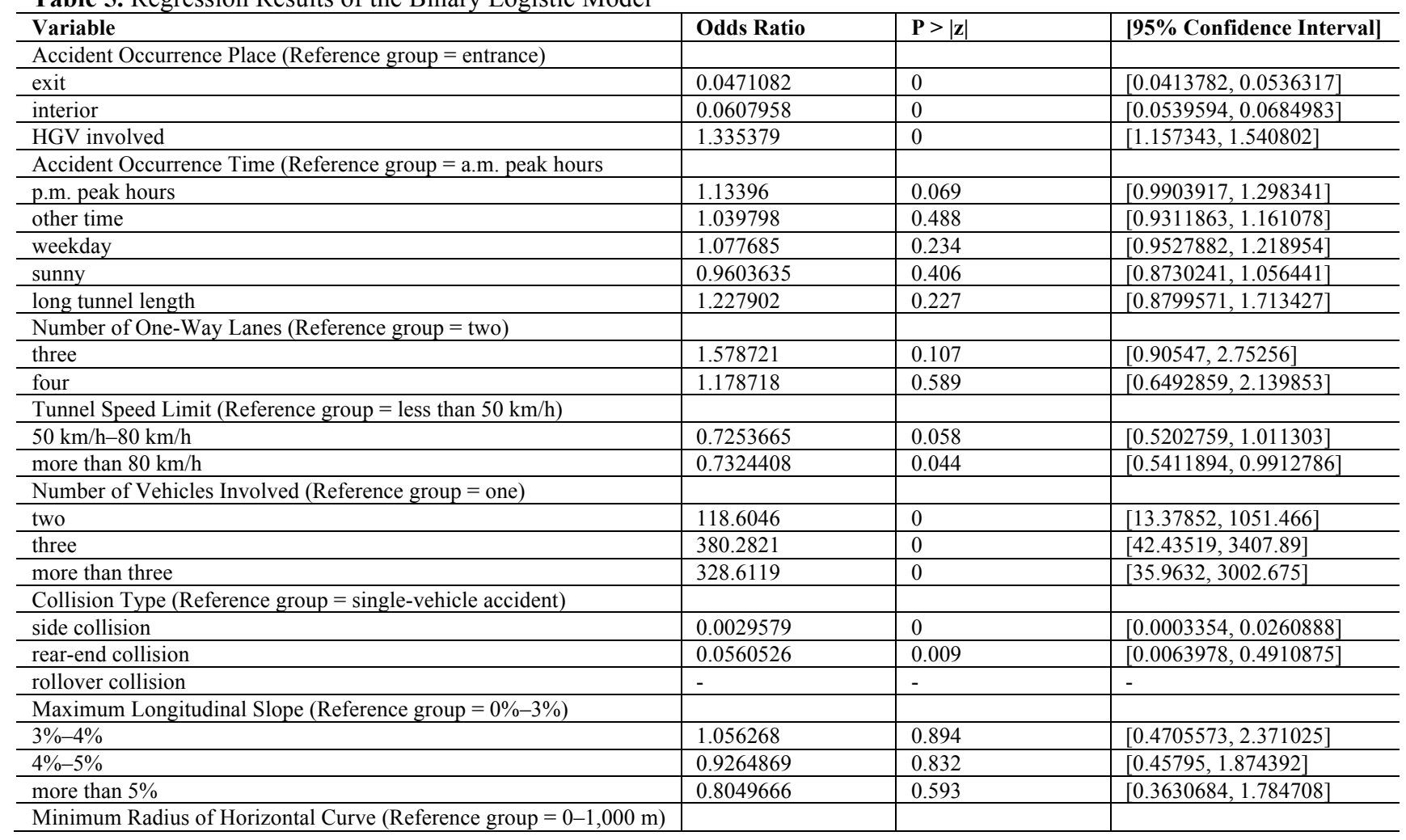




\begin{tabular}{l|l|l|l}
$1,000-2,000 \mathrm{~m}$ & 1.175413 & 0.24 & {$[0.8974763,1.539422]$} \\
\hline $3,000-4,000 \mathrm{~m}$ & 1 & & \\
\hline cons & 0.4965509 & 0.108 & \\
\hline LR chi ${ }^{2}(23)$ & 1332.00 & & \\
\hline Prob $>$ chi $^{2}$ & 0.0000 & & \\
\hline Pseudo ${ }^{2}$ & 0.0797 & \\
\hline
\end{tabular}

(1) Accident-involved vehicle type

Relative to the accident with only light vehicles involved, the occurrence probability of a severe accident can increase by $33.5 \%$ if a truck is involved in the accident. Oversize vehicles have a great impact force with difficult braking, which can post a threat to other vehicles on the road, thereby causing a severe accident. A traffic control strategy should thus be adopted for heavy vehicles during the peak period to control the proportion of heavy vehicles. Doing so can also reduce the occurrence risks of severe accidents in urban river-crossing tunnels.

\section{(2) Accident occurrence place}

The number of accidents at the tunnel entrance, which accounts for $46.5 \%$ of the total data, is higher than those inside the tunnel and at the tunnel exit. Regression results show that if the accident occurs at the tunnel entrance, then the greatest influence on accident severity can be exerted. Moreover, the occurrence probability of severe accidents is 15 and 20 times of those inside urban river-crossing tunnels and at their exit, respectively. Given the complicated linear combination and "black hole effect" at the entrance of urban river-crossing tunnels, controlling vehicles when entering such tunnels become difficult for drivers, easily causing severe accidents. For the longitudinal slope and "black hole effect" at the tunnel entrance, this study provides the following suggestions. Longitudinal slopes should be as small as possible on the precondition that the construction standard is met in the design of urban river-crossing tunnels. Illuminating brightness in the transition section of the tunnel entrance should also be properly adjusted to relieve the influence of the "black hole effect." Conspicuous warning signs should likewise be set at the entrance to raise drivers' vigilance so they can drive carefully.

\section{(3) Number of Vehicles Involved}

The greater the number of vehicles involved in an accident, the more severe the accident. Therefore, "safe distance" signs should be set inside urban river-crossing tunnels to remind drivers to keep a certain safe distance from other vehicles.

\section{(4) Accident type}

According to the results shown in Table 4, single-vehicle accidents can generate severe accidents most easily, followed by rear-end and side collisions. Single-vehicle accidents refer to a vehicle colliding with a fixed object because the driver can easily generate a "side-wall effect" when driving in an urban river-crossing tunnel. That is, the side wall in the tunnel can easily impose mental stress on the driver. Moreover, urban river-crossing tunnels have a complicated linear combination, and their horizontal curve is not as smooth and gentle as those in highway tunnels and open roads. Therefore, under the "side-wall effect" and the linear combination (superposition of longitudinal slope and horizontal curve) of urban river-crossing tunnels, singlevehicle accidents can generate severe accidents most easily. Furthermore, the coherence of horizontal alignment should be realized in the design of urban river-crossing tunnels.
Proper width should also be left at two-lane sides in such tunnels.

\section{(5) Speed limit}

Speed is always an important hidden danger in accidents. Results show that relative to the speed limit below $50 \mathrm{~km} / \mathrm{h}$, the occurrence probability for severe accidents can be reduced by $27 \%$ and $26 \%$ under the speed limit of 50 $80 \mathrm{~km} / \mathrm{h}$ and beyond $80 \mathrm{~km} / \mathrm{h}$ in urban river-crossing tunnels, respectively. As such tunnels do not have a signal lamp, vehicles can easily speed up when the speed limit is too low, resulting in great speed fluctuation inside urban rivercrossing tunnels and increased occurrence rate of accidents. Accident severity can be intensified under evident vehicle speed difference. If the speed limit is slightly up-regulated, then the speed difference will be small, traffic flow will be stable, and collisions will rarely occur. Therefore, the speed limit of urban river-crossing tunnels should be dynamically controlled. Low speed limit can be used under a large traffic volume. If the traffic volume is not large, then the speed limit can be properly elevated.

The binary logistic model can be used to obtain comprehensive influence factors on accident severity by multiplying these factors mutually. Through an experiment on the joint influences of various factors, certain results of significant influence are obtained (Table 6). Results show that if a truck is involved in a rear-end and side collision, then the occurrence probability of severe accidents can increase. If an accident occurs in an urban river-crossing tunnel at morning peak or the number of vehicles involved exceeds three, then the probability can also increase.

Table 6. Cross-Significant Impact Indicator for Binary Logistic Model

\begin{tabular}{l|l|l}
\hline Accident Severity & Odds Ratio & $\mathbf{P}>|\mathbf{Z}|$ \\
\hline HGV involved* side collision & 1.5875458 & 0.009 \\
HGV involved* rear-end collision & 0.6787797 & 0.014 \\
a.m. peak hours * inside the tunnel & 0.7549648 & 0.006 \\
other time * inside the tunnel & 1.204778 & 0.046 \\
three vehicles involved * inside the tunnel & 3.155656 & 0.000 \\
cons & 0.3530972 & 0.000 \\
\hline
\end{tabular}

\subsection{Analysis of simulation modeling results}

Given the restricted original accident data, which were not included in the traffic volume, VISSIM and SSAM were used to further study the influence of traffic volume on accident severity. Selected influence factors were traffic volume, tunnel speed limit, tunnel longitudinal slope, and ratio of heavy trucks among the three traffic elements. Different test scenes related to four factors with five levels were designed through the orthogonal test array. VISSIM was used for simulation. Then, traveling trajectories of vehicles were obtained and imported to SSAM to obtain the number of traffic conflicts. This attribute is an alternative index for studying accident severity. The greater the number of traffic conflicts, the more easily it can generate influences on accident severity.

The range and variance analysis results of the orthogonal test are shown in Fig. 2 and Table 7, respectively. Fig. 2 
illustrates that traffic volume has the greatest influence on the number of traffic conflicts, followed by tunnel longitudinal slope, ratio of heavy trucks, and speed in succession. Similarly, Table 7 reveals that traffic volume has a very significant influence on the number of traffic conflicts and tunnel longitudinal slope. However, the speed and ratio of heavy trucks have relatively small influences. The safest and the most dangerous combination of the four factors can be obtained as well. The safest combination is $1,000 \mathrm{vph}$ (traffic volume), 4\% longitudinal slope, $0 \%$ ratio of heavy trucks, and $80 \mathrm{~km} / \mathrm{h}$ speed limit. The most dangerous combination is $3,000 \mathrm{vph}$ (traffic volume), $6 \%$ longitudinal slope, $10 \%$ ratio of heavy trucks, and $40 \mathrm{~km} / \mathrm{h}$ speed limit. Considering such results, dynamic traffic flow control should be performed in urban river-crossing tunnels. When the traffic volume reaches a saturation status, drivers may choose another route. Longitudinal slopes should be reduced in the design of urban river-crossing tunnels. The distance of such slopes should also be enlarged so that the driving process can be gentle and stable.

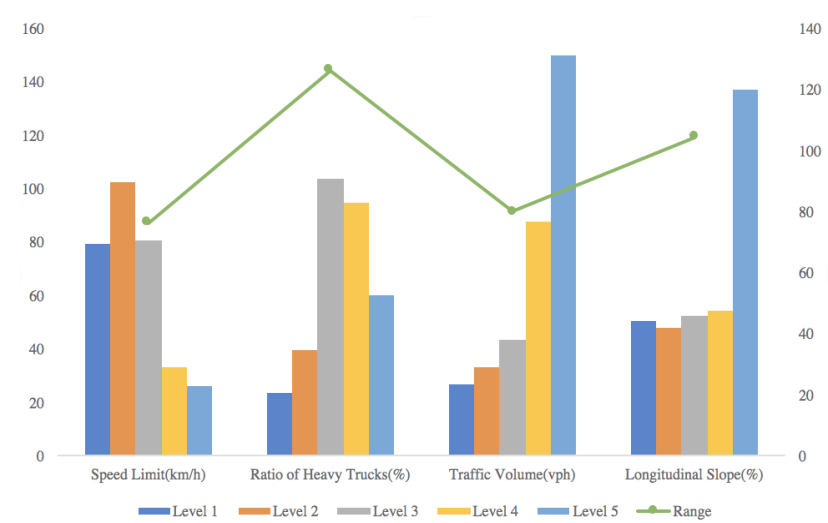

Fig. 2. Range analysis results of the Orthogonal Test

Table 7. Variance Analysis Results of the Orthogonal Test

\begin{tabular}{l|l|l|l|l|l}
\hline Indicator & $\begin{array}{l}\text { Degree } \\
\text { Freedom }\end{array}$ & $\begin{array}{l}\text { Adj } \\
\text { Stdevsquare }\end{array}$ & $\begin{array}{l}\text { Mdj } \\
\text { Meansquare. }\end{array}$ & F-Value & $\begin{array}{l}\text { P- } \\
\text { Value }\end{array}$ \\
$\begin{array}{l}\text { Speed Limit } \\
\text { (km/h) }\end{array}$ & 4 & 22,053 & 5513 & 2.93 & 0.091 \\
$\begin{array}{l}\text { Ratio of } \\
\text { Heavy }\end{array}$ & 4 & 23,980 & 5995 & 3.19 & 0.076 \\
$\begin{array}{l}\text { Trucks (\%) } \\
\text { Traffic Flow }\end{array}$ & 4 & 59,784 & 14946 & 7.95 & 0.007 \\
(vph) & & 34,332 & 8583 & 4.57 & 0.033 \\
$\begin{array}{l}\text { Longitudinal } \\
\text { Slope (\%) }\end{array}$ & 4 & 15,039 & 1880 & & \\
Error & 8 & 155,188 & & & \\
Total & 24 & & & &
\end{tabular}

\section{Conclusions}

As for the influence factors of accident severity in urban river-crossing tunnels, the binary logistic model was used to directly analyze influence relations and influence degrees of various factors on accident severity. The joint influences of every two factors on accident severity were also analyzed based on the historical accident data. In addition, VISSIM and SSAM were used to indirectly analyze the influence factors of accident severity. The following conclusions were drawn.

(1) The significant influence factors of accident severity in urban river-crossing tunnels are traffic volume, number of vehicles involved, accident occurrence place, accident type, tunnel longitudinal slope, tunnel speed limit, and accidentinvolved vehicle type. Among these factors, traffic volume, number of vehicles involved, accident occurrence place, and accident type have the greatest influences on accident severity, followed by tunnel longitudinal slope, tunnel speed limit, and accident-involved vehicle type.

(2) The accident severity of urban river-crossing tunnels is considered the dependent variable. A binary logistic model is also established to study influence factors. Results show that the model has a favorable degree of fitting and can thus analyze the accident severity of urban river-crossing tunnels. Moreover, improvement measures are proposed.

(3) The simulation can be used to indirectly analyze the influence factors of accident severity by changing the driving environment in urban river-crossing tunnels. The simulation method can be combined with the logistic regression model to study accident severity.

To investigate the influence factors of accident severity in urban river-crossing tunnels, mathematical statistical analysis and simulation are combined in this study, making the research comprehensive. However, further research on the combinational features of horizontal curves and longitudinal slopes of urban river-crossing tunnels, as well as interactions among factors in the orthogonal test array, must be conducted.

\section{Acknowledgements}

This work was supported by the National Science Foundation of China (NSFC grant no. E080701 / 51508325).

This is an Open Access article distributed under the terms of the Creative Commons Attribution License

\section{References}

1. John, L. J., Xing, Y., Wang, C., "Risk Factors Affecting the Severity of Traffic Accidents at Shanghai River-crossing Tunnel". Traffic Injury Prevention, 17(2), 2016, pp.176-180.

2. Xing, Y., Lu, J., Wang, C., "Single Vehicle Traffic Accidents in Shanghai River-crossing Tunnels". In: Information Technology and Mechatronics Engineering Conference, Chongqing, China: IEEE, 2015, pp.219-225.

3. Jiang, C., Lu, J. J., Lu, L., "Analysis of Single-Vehicle Crash Injury Severities in Urban River-Crossing Road Tunnels". Applied Mechanics \& Materials, 743(11), 2015, pp.526-532.

4. Caliendo, C., De, Guglielmo. M. L., Guida, M., "A crash-prediction model for road tunnels". Accident Analysis \& Prevention, 55, 2013, pp. 107-115.
5. Calvi, A., De, Blasiis, M. R., Guattari, C., "An empirical study of the effects of road tunnel on driving performance". Procedia-Social and Behavioral Sciences, 53, 2012, pp.1099-1109.

6. McLaughlin, S. B., Hankey, J. M., Klauer, S. G., "Contributing factors to run-off-road crashes and near-crashes". Fatigue, 144(1), 2009, doi: 10.1061/JTEPBS.0000104.

7. Liu, C., Subramanian, R., "Factors related to fatal single-vehicle runoff-road crashes (Tech. Rep. No. DOT HS 811 232)". Washington, United States: NHTSA, 2009.

8. Costescu, D., Raicu, S., Rosca, M., "Using intersection conflict index in urban traffic risk evaluation". Procedia Technology, 22, 2016, pp. 319-326. 
9. Autey, J., Sayed, T., Zaki, M. H., "Safety evaluation of right-turn smart channels using automated traffic conflict analysis". Accident Analysis \& Prevention, 45, 2012, pp.120-130.

10. Amundsen, F. H., Ranes, G., "Studies on traffic accidents in Norwegian road tunnels". Tunnelling \& Underground Space Technology Incorporating Trenchless Technology Research, 15(1), 2000, pp.3-11.

11. Mashimo, H., "State of the road tunnel safety technology in Japan". Tunnelling \& Underground Space Technology Incorporating Trenchless Technology Research, 17(2), 2002, pp.145-152.

12. Lemke, K., "Road safety in tunnels". Transportation Research Record: Journal of the Transportation Research Board, 1740 , 2000, pp. 170-174.

13. Nussbaumer, C., "Comparative analysis of safety in tunnels". In: Young Researchers Seminar, Brno, Czech Republic: TTIC, 2007, pp. 27-30.

14. Sam, E. F., Daniels S., Brijs K., Brijs, T., Wets G., "Modelling public bus/minibus transport accident severity in Ghana". In: International Co-Operation on Theories and Concepts in Traffic Safety, Olomouc, Czech Republic: ICTCT, 2017, pp.114-121.
15. Potoglou, D., Carlucci, F., Cirà, A., \& Restaino, M., "Factors associated with urban non-fatal road-accident severity". International Journal of Injury Control \& Safety Promotion, 25(3), 2018, pp.303-310.

16. Ahmed, M. M., Franke, R., Ksaibati, K., "Effects of truck traffic on crash injury severity on rural highways in Wyoming using Bayesian binary logit models". Accid Anal Prev, 117, 2018, pp.106-113.

17. Haynes, R., Lake, I. R., Kingham, S., Sabel, C. E., Pearce, J., Barnett, R., "The influence of road curvature on fatal crashes in new Zealand". Accid Anal Prev, 40(3), 2008, pp.843-850.

18. Jung, S., Qin, X., Noyce, D. A., "Modeling Highway Safety and Simulation in Rainy Weather". Transportation Research Record Journal of the Transportation Research Board, 2237(2237), 2011, pp.134-143.

19. Simončič, M., "Road accidents in Slovenia involving a pedestrian, cyclist or motorcyclist and a car". Accident Analysis \& Prevention, 33(2), 2001, pp.147-156.

20. Jiang, C., Lu, L., Chen, S., Lu, J. J., "Hit-and-run crashes in urban river-crossing road tunnels". Accident Analysis \& Prevention, 95 2015, pp.373-380.

21. Chen, G., Chen, D., "Traffic safety analysis of tunnel entrance section of mountainous expressway based on VISSIM". Jiangxi Building Materials, 22, 2014, pp.178-180. (In Chinese) 